\title{
Pierre BRÉCHON, Frédéric GONTHIER (dir.), 2015, Atlas des Européens. Valeurs communes et différences nationales, Paris, Armand Colin, 128 p.
}

Olivia Leboyer

\section{OpenEdition}

Journals

Édition électronique

URL : http://journals.openedition.org/ress/2966

DOI : $10.4000 /$ ress. 2966

ISSN : 1663-4446

Éditeur

Librairie Droz

Édition imprimée

Date de publication : 15 mai 2015

Pagination : 306-308

ISSN : 0048-8046

Référence électronique

Olivia Leboyer, «Pierre BRÉCHON, Frédéric GONTHIER (dir.), 2015, Atlas des Européens. Valeurs communes et différences nationales, Paris, Armand Colin, 128 p. », Revue européenne des sciences sociales [En ligne], 53-1 | 2015, mis en ligne le 13 février 2015, consulté le 22 septembre 2020. URL http://journals.openedition.org/ress/2966 ; DOI : https://doi.org/10.4000/ress.2966

Ce document a été généré automatiquement le 22 septembre 2020.

(C) Librairie Droz 


\title{
Pierre BRÉCHON, Frédéric GONTHIER (dir.), 2015, Atlas des
} Européens. Valeurs communes et différences nationales, Paris, Armand Colin, $128 \mathrm{p}$.

\author{
Olivia Leboyer
}

1 Comment saisir, au plus juste, l'Europe ? Peut-on mettre au jour un principe d'unité qui relierait ces vingt-sept pays? Sans doute pas. Sur le plan géographique comme sur le plan politique, la construction européenne n'a pas atteint une forme définitive. Peut-on alors, déterminer un ensemble de valeurs européennes, qui correspondrait à un modèle commun? Plus prudemment, les directeurs de l'ouvrage ont choisi pour titre Atlas des Européens afin de souligner le pluralisme des valeurs et la tendance à l'individualisation. Dans le sous-titre Valeurs communes et différences nationales les idées d'unité et de diversité se croisent ainsi en chiasme. Pour représenter les évolutions de cet espace, la cartographie apporte un éclairage utile. Depuis trente ans, la European Values Study s'attache à sonder les mentalités des Européens, au fil d'une enquête qui tente de couvrir tous les domaines de l'existence. Si l'ambition est immense, les chercheurs se sont donné les moyens de la réaliser : il s'agit, en effet, de collecter et d'interpréter les résultats de sondages méthodiques, réitérés tous les neuf ans, dans quarante-six pays européens. On le voit, c'est ici une Europe au sens large, proche de celle du Conseil de l'Europe, qui est examinée. L'enquête ne part pas d'une idée de l'Europe, connue et établie, mais bien d'une interrogation sur ce qu'est l'Europe.

Soutenu par le laboratoire PACTE de Grenoble, ce travail a été mené à bien par deux cartographes, Isabelle André-Poyaud et Nicolas Robinet, et toute une équipe de spécialistes de science politique, sociologie et géographie. Sept grands champs thématiques ont été sélectionnés : identités et appartenances; bonheur individuel et lien social; éthique individuelle et normes sociales; famille; travail et économie; 
religion ; politique - avec un nombre conséquent de notices assorties d'un commentaire synthétique. Il y a là un désir d'exhaustivité, Pierre Bréchon et Frédéric Gonthier n'hésitant pas à dire que l'atlas couvre tous les domaines de la vie humaine.

L'impression de simplicité domine, les grandes questions posées ayant nécessairement quelque chose de schématique: "Tolérez-vous l'incivisme?", "Accordez-vous plus d'importance à votre travail ou à votre vie de famille?", "Êtes-vous optimiste?", "Avez-vous confiance en autrui ?». On sait, au moins depuis Pierre Bourdieu, à quel point de vastes questions, posées frontalement, peuvent induire des réponses formatées, presque mécaniques. Une personne interrogée aura certainement tendance à répondre de manière plutôt consensuelle sur des questions comme "Vous sentezvous solidaire avec autrui ?", "Avez-vous des principes éthiques? » ou "Respectezvous les autres?». De même, pour les valeurs à transmettre aux enfants, le partage entre les valeurs matérialiste et les valeurs altruiste parait, pour le moins, sommaire. Mais certaines questions, plus insolites, entraînent peut-être davantage de confidences. Ainsi, la question «Choisir ses voisins : qui veut-on éviter?" permet à un racisme latent ou à un sentiment d'insécurité de s'exprimer plus ouvertement. De même, «Croyez-vous aux porte-bonheur?» met l'accent sur les nouvelles formes de religiosité, plus diffuses et hors des pratiques collectives.

Les cartes, avec leurs couleurs vives et leurs hachures, se lisent plutôt facilement. Le plus souvent, seules deux variables sont mises en corrélation. Dans certaines cartes, il n'est pas évident de discerner la taille des hachures. Mais, dans l'ensemble, elles sont établies avec une grande clarté. Dès lors, qu'apprend-on de nouveau ? Tout l'intérêt de ces cartes tient, sans doute, aux différents niveaux de lecture qu'elles offrent. Au premier coup d'œil, c'est souvent une impression d'évidence qui frappe. Des énoncés comme "On tend à se sentir plus heureux dans les pays riches. On y est aussi plus souvent satisfait de la manière dont la démocratie fonctionne » ou «Plus les actifs d'un pays ont le sentiment d'être libres dans leur travail, et plus ils en sont satisfaits » sonnent un peu comme des lapalissades. Mais, en y regardant de plus près, les nuances entre les pays peuvent étonner. Ainsi, les pays où les habitants sont plus satisfaits de leur travail qu'attendu ne sont guère proches géographiquement et n'ont pas de point commun apparent: Islande, Suisse, Chypre, Irlande, Kosovo. À l'inverse, les pays où les habitants se disent les moins satisfaits ont une proximité géographique et, peut-être, un trait culturel commun : Arménie, Azerbaïdjan, Géorgie, Albanie, Turquie.

5 Pour chaque carte, l'analyse semble à la fois logique et déroutante, certaines exceptions ne s'expliquant pas aisément. Par exemple, pour les pays demandeurs de plus d'autorité, et qui ont donc davantage confiance dans l'armée et la police, les différences ne sont pas faciles à analyser. S'il paraît logique que les pays de l'ex-bloc de l'Est éprouvent peu de confiance dans les autorités militaires, en raison de leur histoire, la forte demande de la part de la Belgique, des Pays-Bas, du Danemark ou de l'Italie ne répond pas à une urgence particulière.

6 Soulignons la pertinence de certains questionnements, plus originaux : «Préférez-vous la liberté ou l'égalité ? ", avec $55 \%$ en faveur de la liberté et $45 \%$ en faveur de l'égalité, met l'accent sur une tension fondamentale pour la démocratie libérale. D'une manière générale, les citoyens préfèrent ce qui leur manque. L'égalité est alors très peu choisie à la fois dans les pays les plus égalitaires et dans les plus inégalitaires. Dans le cas des pays de l'Est, où le processus de transition démocratique est récent, on remarque une difficulté à se situer politiquement sur l'axe gauche-droite. Dans un tout autre domaine, 
la famille, la question des devoirs réciproques entre parents et enfants constitue un angle intéressant. À l'intérieur du thème "Éthique individuelle et normes sociales ", l'enquête sur les attitudes vis-à-vis des biotechnologies permet également de poser un dilemme important.

7 Chacun des sept thèmes comprend ainsi des analyses assez générales, sans grande surprise, et d'autres plus inattendues, qui apportent un éclairage différent. Dès l'introduction, les directeurs de l'atlas insistent sur la grande liberté du lecteur, qui peut «naviguer à sa guise» d'une question à l'autre. Pas de clef de lecture, ou de méthode interprétative imposée: les valeurs des Européens apparaissent ici sous la forme d'une mosaïque ou d'un kaléidoscope, à la fois familières et étranges. C'est l'intelligence du lecteur qui est sollicitée. En croisant ensemble plusieurs cartes, dans la même thématique ou non, il est possible d'enrichir les analyses proposées, qui se présentent comme des pistes de réflexion. Selon sa curiosité, le lecteur peut considérer plus attentivement tel pays en particulier, ou bien un groupe de pays. Synthétique, maniable, l'atlas se veut un guide souple, non directif, que l'on peut survoler ou bien utiliser avec sérieux et méthode.

8 Si l'usage de l'atlas est libre, Pierre Bréchon et Frédéric Gonthier entendent tout de même tirer quelques enseignements de l'enquête menée et proposent une courte conclusion de deux pages. Quelques constatations s'imposent: les valeurs des Européens ne forment pas un ensemble homogène et lisse, mais se caractérisent par leur diversité ; les valeurs d'un pays sont souvent le fruit de son histoire, la lecture des cartes supposant des connaissances; les liens entre certaines valeurs, comme le libéralisme économique et la libéralisation des mœurs, ne sont pas automatiques; la proximité géographique n'implique pas nécessairement des valeurs communes. Il va de soi que les cartes et notices ne constituent pas une vérité intangible, les cultures nationales de chaque pays étant évidemment elles-mêmes plurielles. On peut aussi s'interroger sur le choix d'un découpage par pays, alors que l'on aurait pu, tout aussi bien, comparer utilement tel Land allemand avec telle région française, par exemple. Mais les contraintes choisies ont leur avantage, celui de la lisibilité.

9 Au terme de l'ouvrage, on peut distinguer schématiquement une Europe du Nord d'une Europe du Sud, où la participation politique et la politisation sont plus faibles, où la confiance en autrui et dans les institutions est moins affirmée. Cet axe se comprend selon une distinction religieuse entre une Europe davantage protestante au Nord et une Europe majoritairement catholique au Sud. De la même façon, il ressort qu'en Europe occidentale, les citoyens tendent moins à s'identifier à leur pays, tandis que la demande d'ordre augmente. À l'Est, la méfiance semble plus prégnante. Dans toute cette aire européenne, au sens large, la tendance à l'individualisation des valeurs constitue un fait majeur, ce mouvement ne se confondant pas avec l'individualisme au sens étroit. En effet, les formes de sociabilités observées témoignent d'un attachement fort, dans tous les pays, aux liens sociaux. 


\section{AUTEURS}

OLIVIA LEBOYER

IEP de Paris- PACTE 